\title{
Management of refractory septic shock. Can intra-aortic balloon counterpulsation be the answer?
}

\author{
Gentle Sunder Shrestha ${ }^{*}$, Gaurav Nepal ${ }^{2}$ \\ 'Department of Anaesthesiology; ${ }^{2}$ Department of Internal Medicine, Tribhuvan University Teaching \\ Hospital, Maharajgunj, Kathmandu, Nepal
}

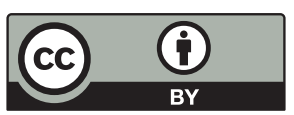

This work is licensed under a Creative

Commons Attribution 4.0 Unported License.

\begin{abstract}
Refractory septic shock (RSS) is a subset of septic shock that does not respond to standard resuscitation and to high-dose vasopressor therapy. Management of RSS remains challenging and is associated with increased mortality. There is a dire need to identify a novel therapeutic strategy for the management of RSS. Extracorporeal membrane oxygenation (ECMO) and intra-aortic balloon counterpulsation (IABP) are potential options for the management of RSS. Since ECMO is expensive, requires high expertise, and has more complications, IABP can be a feasible therapeutic option. IABP may help clinicians buy the time by supporting the organ perfusion while treating sepsis. Judicious patient selection can be the key. Well designed clinical trials may be the way forward.
\end{abstract}

\section{KEY WORDS}

Intraaortic balloon counterpulsation, refractory septic shock.

\section{INTRODUCTION}

Refractory septic shock (RSS) is a subset of septic shock that does not respond to standard resuscitation and is associated with very poor outcome.1,2 There is no universal consensus definition of refractory shock. However, it can be defined as septic shock with inadequate response to high-dose vasopressor therapy (> $0.5 \mathrm{mcg} / \mathrm{kg} / \mathrm{min}$ norepinephrine or equivalent). ${ }^{2}$ Nearly $6-7 \%$ of critically ill patients in the intensive care unit (ICU) develop RSS, which account for $18 \%$ of ICU deaths. The mortality rate of RSS patients is as high as $60 \%{ }^{2,3}$

Management of refractory septic shock remains challenging. Treatment of refractory septic shock usually requires long-term use of high doses of catecholamines, but this high-dose use is associated with increased ICU mortality caused by peripheral and splanchnic ischemia, acute myocardial infarction, atrial fibrillation, increased oxygen consumption and hyperglycemia. ${ }^{4}$ Hydrocortisone, Vitamin C, thiamine, methylene blue, hydroxocobalamin, vasopressin and angiotensin $\|$ have been studied previously but they failed to show promising effects. None

\footnotetext{
*Corresponding Author

Dr. Gentle Sunder Shrestha

Department of Anaesthesiology, Tribhuvan University Teaching Hospital

Maharajgunj, Kathmandu, Nepal

Email: gentlesunder@hotmail.com

Phone: +977-9841248584
} 
of the above agents have been extensively studied in RSS. ${ }^{1,4}$ In randomized controlled trials investigating the effects of various interventions on septic shock, this subgroup of patients is often underrepresented. ${ }^{2}$ As a result, there is hardly any conclusive evidence to guide the management of septic shock in this particular group of people. Therefore, there is dire need to identify novel therapeutic strategy for RSS management.

\section{CURRENT UNDERSTANDING}

Little is known about the pathophysiology of RSS. It is hypothesized that septic cardiomyopathy caused by chemical mediators such as endotoxin, cytokines and nitric oxide alters myocardial structure and function, resulting in insufficient preload, decreased ejection fraction, poor left ventricular dilatation and myocardial contractility. ${ }^{5}$ Therefore, to deal with RSS, we need to hobnob with myocardial function. High dose vasopressors alone can't maintain myocardial function and hemodynamics in RSS. ${ }^{\text {? }}$ Other effective options to maintain these are judicial use of extracorporeal membrane oxygenation (ECMO) and intraaortic balloon counterpulsation (IABP). However, ECMO is too intrusive, expensive, requires high expertise, and is accompanied by numerous complications. In addition to all the shortcomings, the high level of expertise and the myriad of complications are the main limitations of using ECMO in RSS patients. ${ }^{5}$ Therefore, IABP can be a better option to support hemodynamics in RSS. IABP reduces the afterload and promotes forward flow of the blood during systole. Also, IABP increasing coronary blood flow during diastole, and thus potentially improving myocardial contractility. These mechanisms may help the myocardium that is compromised due to sepsis and is being whipped with high dose of inotropes and vasopressors. IABP may serve as a bridge to support hemodynamics, while buying time for antibiotics to work effectively and the failing organs to recover.

A study from china showed that IABP when combined with dopamine and norepinephrine can significantly improve hemodynamics, increase coronary and systemic tissue perfusion, reduce cardiac afterload, elevate cardiac index, reduce doses of vasoactive drugs, shorten length of ICU stay, improve prognosis, and lower the mortality rate than dopamine and norepinephrine alone. ${ }^{6}$ Similarly, a study from Czech Republic with 10 subjects found that after 60 hours of balloon counterpulsation, there was a statistically significant increase in MAP at concomitant reduction in catecholamine doses. At the end of the reporting period, $90 \%$ succeeded achieve the predefined MAP level (above 65 $\mathrm{mm} \mathrm{Hg}$ ). They also observed that IABP can improve renal perfusion and improve renal function. ${ }^{7}$ In a Japanese case series including two cases in whom, despite conventional therapy, shock was not reversed, after insertion of IABP, circulatory status was improved. They concluded that IABP can be used to rescue patients with septic cardiomyopathy who have refractory septic shock. ${ }^{8}$ In a case report from Canada, IABP successfully improved circulation in a $76 \mathrm{yr}$ old hypodynamic septic shock patient to the point at which the patient could maintain adequate cardiac output without assistance. ${ }^{9}$ Similarly, a case report from Taiwan showed that use of IABP improved volume and inotrope refractory shock over a period of 3 days in patient with meningococcal myocarditis. ${ }^{10}$

A case report from Singapore showed similar efficacy. A 78-year-old female developed worsening shock, sepsisinduced cardiomyopathy and multi-organ failure, leading to an episode of pulseless electrical activity. But after insertion of IABP, patient's condition improved dramatically. She was weaned off from circulatory supports, and eventually discharged home. ${ }^{11}$ Another case report from Singapore reported a case of intestinal tuberculosis induced septic shock with septic cardiomyopathy. She underwent surgery for tuberculous peritonitis and intestinal perforation. Later she developed septic shock with septic cardiomyopathy unresponsive to vasopressor and inotrope. IABP was inserted and there was drastic haemodynamic improvement. She continued to improve, and was weaned off from circulatory supports, and eventually transferred to the general ward. ${ }^{12}$ Similarly, a case report from Washington reported a case of successful IABP use in a patient with septic shock and severe septic cardiomyopathy in a 28-year-old African American female who underwent dilation and curettage for incomplete, septic abortion. She was admitted to the ICU with septic shock, and her condition deteriorated overnight with escalation of vasopressor doses, short runs of ventricular tachycardia, ARDS and renal failure. Despite the addition of epinephrine and dobutamine, shock remained refractory. Decision was made to utilize IABP and within couple of hours after IABP initiation, hemodynamics, cardiac index and mixed venous oxygen saturation improved, arrhythmias subsided, and urine output increased. She was weaned off from circulatory supports, and eventually discharged well. ${ }^{13}$

The initial widespread use of IABP for management of patients with cardiogenic shock secondary to acute myocardial infarction was shadowed after the publication of IABP-SHOCK II trial, which showed no benefit of IABP for reduction of 30 days mortality. Of note, they enrolled patients undergoing early coronary revascularization. ${ }^{14}$ RSS carries higher mortality than cardiogenic shock. Patients with cardiogenic shock are usually older and with multiple comorbidities. Septic shock can affect patients of any 
age, from newborn to elderly. A recent global burden of diseases study revealed that the actual estimates of cases of sepsis and septic shock are more than double the previous picture and preferentially affects the population in low and middle income nations and in a younger age group. ${ }^{15}$

\section{WAYS FORWARD}

As with many interventions in critical care, likely not all patients with RSS would benefit with IABP. The "one size fits all" approach of multiple large trials in the past have failed to show reasonable benefits. ${ }^{16}$ Judicious patient selection for this intervention, like those with treatable source of infection and those without established irreversible organ dysfunction, may benefit with IABP. A well designed clinical trial of IABP in RSS, which incorporates precision medicine may be a way ahead to explore the possible benefit of this intervention. ${ }^{17}$ Embracing adaptive trial design may add flexibility and efficiency. $^{18}$

\section{CONFLICTS OF INTEREST: None}

\section{REFERENCES}

1. Jentzer JC, Vallabhajosyula $S$, Khanna AK, Chawla LS, Busse LW, Kashani KB. Management of Refractory Vasodilatory Shock. Chest. 2018;154(2):41626.

2. Nandhabalan P, loannou N, Meadows C, Wyncoll D. Refractory septic shock: our pragmatic approach. Crit Care. 2018;22(1):215.

3. Bassi E, Park M, Azevedo LCP. Therapeutic Strategies for High-Dose Vasopressor-Dependent Shock. Crit Care Res Pract. 2013;2013:1-10.

4. Antonucci E, Gleeson PJ, Annoni F, Agosta S, Orlando S, Taccone FS, et al. Angiotensin II in Refractory Septic Shock. Shock. 2017;47(5):560-6.

5. Sato R, Nasu M. A review of sepsis-induced cardiomyopathy. J Intensive Care. 2015;3(1):1-7.

6. Wei C, Sheng B, Zhao L, Lu F, Wang S, Liu L, et al. The clinical application and value of intra-aortic balloon pump in patients with septic shock. Chin Crit Care Med. 2012;24(1):46-9.

7. Ondrášková H. Influence of Intraaortal Balloon Counterpulsation ON Renal Function During Septic Shock. Masaryk University in Brno; 2008.

8. Hashiba K, Hiromi T, Nakamura K, Toida C, Morimura $\mathrm{N}$, Doi $\mathrm{T}$, et al. Two cases with intra-aortic balloon pumping use for severe septic cardiomyopathy. Acute Med Surg. 2017;4(4):446-50.

9. Mercer D, Doris P, Salerno TA. Intra-aortic balloon counterpulsation in septic shock. Can J Surg. 1981;24(6):6435.

10. Chiu YH, How CK, Chern $\mathrm{CH}$, Wang LM, Huang $\mathrm{Cl}$. Cardiac rescue with intra-aortic balloon counterpulsation in refractory shock due to acute meningococcemia. Am J Emerg Med. 2007;25(2):253-4.

11. Dalakoti M, See KC. Successful use of intra-aortic balloon pump in sepsis-induced cardiomyopathy: A case report. IHJ Cardiovasc Case Reports. 2018;2(3):164-5.

12. Ng E-L, Yeo L, Siddiqui S. Intra-aortic balloon pump as supportive treatment for sepsis-induced cardiomyopathy and cardiogenic shock in a patient with intestinal tuberculosis. In: Euroanesthesia 2018. 2018. p. 03.

13. Sokolovic M LB. Succesful Useof IABP In Patient With Septic Shock And Septic Cardiomyopathy. In: Critical Care Medicine. 2012. p. 1-138.

14. Thiele $H$, Zeymer $U$, Neumann $F$, Ferenc $M$, Olbrich $\mathrm{H}$, Hausleiter J, et al. Intraaortic balloon support for myocardial infarction with cardiogenic shock. N Engl J Med. 2012;367(14):1287-96.

15. Rudd KE, Johnson SC, Agesa KM, Shackelford KA, Tsoi D, Kievlan DR, et al. Global, regional, and national sepsis incidence and mortality, 1990-2017: analysis for the Global Burden of Disease Study. Lancet. 2020;395(10219):200-11.

16. Santacruz CA, Pereira AJ, Celis E, Vincent JL. Which multicenter randomized trials in critical care medicine have showen reduced mortality? A systematic review. Crit Care Med. 2019;47(12):1680-91.

17. Wong HR, Atkinson SJ, Cvijanovich NZ, Anas N, Allen GL, Thomas NJ, et al. Combining Prognostic and Predictive Enrichment Strategies to Identify Children with Septic Shock Responsive to Corticosteroids. Crit Care Med. 
2016;44(10):e1000-3.

18. Pallmann P, Bedding AW, Choodari-Oskooei B, Dimario M, Flight L, Hampson LV, et al. Adaptive designs in clinical trials: why use them, and how to run and report them. BMC Med. 2018;16(1):29. 